\title{
Minimal standard terminology for gastrointestinal endoscopy - MST 3.0
}

Authors

Institutions
Lars Aabakken, Norway (chairman), Bjorn Rembacken, UK, Olivier LeMoine, Belgium, Konstantin Kuznetsov, Russia, Jean-Francois Rey, France, Thomas Rösch, Germany, Glen Eisen, USA, Peter Cotton, USA, Masayuki Fujino, Japan

OMED Documentation \& Standardisation Committee
Bibliography

DOI $10.1055 / \mathrm{s}-0029-1214949$

Endoscopy 2009; 41 :

727-728 @ Georg Thieme Verlag KG Stuttgart · New York ISSN 0013-726X

\section{Corresponding author}

Lars Aabakken, MD

Department of Medicine

Rikshospitalet University Hospital

Oslo, Norway

Fax: $+43-23072008$

lars.aabakken@medisin.uio.no
Standardization of the language of gastrointestinal endoscopy is becoming increasingly important on account of international collaboration, standardized documentation requirements, and computer-based reporting. Version 1 of the Minimal Standard Terminology (MST) was devised to facilitate this development, and, through broad international collaboration, the document was developed and tested further to produce version 2.0, published in 2000. The document forms the basis for computer software by offering standard minimal lists of terms to be used in the structured documentation of endoscopic findings.The own-

\section{Introduction}

$\nabla$

The widespread implementation of computers for reporting gastrointestinal endoscopy has underlined the importance of a common language, as well as a standardized way of reporting findings. A global collaboration addressing this issue was initiated in 1991 by the European Society for Gastrointestinal Endoscopy (ESGE). Based on the hallmark work of Professor Zdenek Maratka for standardized endoscopic terminology $[1,2]$ this effort involved endoscopy experts from all regions of the world, as well as representatives from the principal manufacturers of endoscopy equipment, and from publishers Normed Verlag who are responsible for printed materials.

The major aim of the project was to devise a "minimal" list of terms that could be included within any computer system used to record the results of a gastrointestinal endoscopic examination. The list was not to be exhaustive, and the work was not to lead to complete software. Rather, the MST was to form a basis for various software vendors, to facilitate a common structure and language. In addition, the MST was to assist in the standardization of endoscopic image storage, in transfer between individual systems, and in structuring reports. ership of the MST has been transferred to the World Organisation of Digestive Endoscopy (OMED) and in this context, a new revision of the MST document is now in place. Version 3.0 of the terminology includes terms for endoscopic ultrasound (EUS) and enteroscopy, as well as for adverse event reporting. In addition, acknowledged scoring systems have been included for specific findings, and some structural enhancements have been implemented.The entire document is freely available for noncommercial use from www.omed.org.

The proposed list of terms relied heavily upon the original and detailed work performed by the OMED committee under the chairmanship and guidance of Professor Zdenek Maratka. His book provides the framework, as well as the definitions for most of the MST terminology, and offers a reference for users unfamiliar with the words employed.

MST $1.0[3,4]$ formed the basis for prospective testing of the terminology in Europe and the United States, which was funded by the European Commission through the GASTER (Gastrointestinal Endoscopy Applications for Standards in Telecommunication, Education, and Research) Project, and by the American Digestive Health Foundation. It relied heavily on the collaboration of Dr. Michel Delvaux on the European and Dr. Louis Korman on the US side. This work resulted in a number of modifications implemented in MST 2.0 in 2000. Since then, this version of the MST has been applied in a number of software solutions, mostly with various modifications. 


\section{MST 3.0}

\section{$\nabla$}

For further development of the MST, the responsibility and copyright were recently transferred to OMED. The Committee for Terminology and Standardization has been in charge of this task, which has resulted in the present version, MST 3.0. While the original ideas of Prof. Maratka, ESGE, ASGE and the GASTER project have been retained, some modifications have been made for this revision.

- Endoscopic ultrasonography (EUS) and enteroscopy (including capsule endoscopy) have been included. The previously published EUS terminology (including definitions) [5] is included in recent versions of Prof. Maratka's book, and forms the basis for the present EUS MST. With regard to enteroscopy, an effort was made to accommodate all endoluminal imaging modalities used to visualize the small bowel, including capsule endoscopy, utilizing common terms and with the same principles of attributes and attribute values as in the rest of the MST document.

- To avoid redundancies and inherent contradictions, the document has been somewhat reorganized, with one generic list of findings for each main category (luminal, ERCP [endoscopic retrograde cholangiopancreatography], EUS). This is coupled with a table to indicate which findings are relevant for which organ. In this way, the description of a polyp remains the same, regardless of the organ involved. This also allows the inclusion of a luminal section in an ERCP reporting software module, with the same structure as for an upper endoscopy.

- The ERCP terminology has been revised to allow more precise description of maneuvers as well as findings.

- Updated internationally recognized classifications have been included as attributes wherever relevant.

- The lists for indications (reasons for endoscopy) and diagnoses have been extended and somewhat revised.

- The section on treatment has been revised and expanded to reflect recent technical developments.

- A new section on reporting of adverse events has been developed, offering description and grading of intraoperative as well as post-procedure events.

The complete MST 3.0 document can be downloaded free of charge from the OMED website (www.omed.org), and is free for use in any commercial or noncommercial context as long as the copyright requirements are honored.

\section{MST and local modifications}

$\nabla$

The discipline of endoscopy is constantly evolving, and performance of endoscopic procedures is quite different between centers, countries and cultures. Thus, although some items of endoscopic language and structure are constant, there is a continuous need for flexibility and customization. This has implications for the MST document. While presenting standards for core items, it should offer, even encourage, such a degree of flexibility that users can accept the inherent structure and limitation that are associated with any standard. Thus, user modification, within the recommendations in the copyright statement, is probably vital to an acceptable role of the MST.

Also, by presenting MST 3.0 in an interactive context on the OMED website, we hope to collect feedback from users that will help to even further improve the document.

The MST offers a selection of terms and attributes for appropriate description of findings, procedures, and complications. It does not offer a complete reference for the endoscopic report. However, in the development of software for endoscopic reporting the MST should be considered as guidance on structure and for initial selection of list terms available.

The relevant local modifications would be:

- adding items to a list

- removing irrelevant items from a list

- adding subclassifications within the main MST items

- adding attributes with corresponding values

\section{Minimal standard documentation}

$\nabla$

While the present MST document offers a template for the main sections of the endoscopy report and standardizes well the descriptions of the findings per se, it is not a complete description of endoscopy documentation. Thus, the OMED committee has set out to develop additional standardizing recommendations to supplement the current MST 3.0 document:

- MSR, or Minimal Standard Reporting. This will offer structure and content recommendations for all the main endoscopic procedures, including formal and technical, as well as medical elements. This document will link closely to current quality control parameters that are presently being implemented in a number of national quality programs.

- MSI, or Minimal Standard Imaging. This will give recommendations for image documentation, for normal procedures (e.g. cecal intubation), as well as for focal and diffuse findings. It is hoped that this will improve the practical output of the imaging features presently available in most commercially available endoscopy reporting softwares.

\section{Competing interests: None}

\section{References}

1 Maratka Z. Terminology, definitions and diagnostic criteria in digestive endoscopy. Scand J Gastroenterol 1984; 19 (Suppl 103): 1-74

2 Maratka Z. Terminology, definitions and diagnostic criteria in digestive endoscopy. 3rd edn. Bad Homburg, Germany: NORMED Verlag, 1994

3 ESGE Committee for Minimal Standards for Terminology and Documentation in Digestive Endoscopy. Minimal standard terminology for databases in digestive endoscopy. Bad Homburg, Germany: NORMED Verlag, 1995

4 Crespi M, Delvaux M, Schapiro $M$ et al. Working Party Report by the Committee for Minimal Standards of Terminology and Documentation in Digestive Endoscopy of the European Society of Gastrointestinal Endoscopy: Minimal standard terminology for a computerized endoscopic database. Am J Gastroenterol 1996; 91: 191-216

5 Aabakken L. Standardized terminology in endoscopic ultrasound. Eur J Ultrasound 1999; 10: 179-183 